\title{
Decay Bounds for Solutions of Second Order Parabolic Problems and Their Derivatives III
}

\author{
L.E. Payne and G.A. Philippin
}

\begin{abstract}
Further extending investigations carried out in previous papers, the authors deal in this paper with another non-linear initial-boundary value problem whose solution, without appropriate data restrictions, might blow up at some finite time. The purpose of this paper is to determine conditions on the geometry and data sufficient to insure that the solution remains bounded and with those conditions to derive exponential decay bounds for the solution and its gradient.
\end{abstract}

Keywords: Quasilinear parabolic problems, maximum principle

MSC 2000: Primary 35K55, secondary 35K60, 35K20

\section{Introduction}

Let $u(\mathbf{x}, t)$ be the classical solution of the initial-boundary value problem

$$
\left\{\begin{aligned}
\nabla(D(\mathbf{x}) \nabla u)-u_{, t} & =-f(u) & & \mathbf{x} \in \Omega, t \in(0, \tau) \\
u(\mathbf{x}, 0) & =g(\mathbf{x}) \geq 0 & & \mathbf{x} \in \Omega \\
u(\mathbf{x}, t) & =0 & & \mathbf{x} \in \partial \Omega
\end{aligned}\right.
$$

supposed to exist for $t \in(0, \tau)$. In (1), $\Omega$ is a bounded convex domain in $\mathbb{R}^{N}, N \geq 2$, and $f, g, D$ are given nonnegative functions. Clearly we have $u(\mathbf{x}, t) \geq 0$ in $\Omega \times(0, \tau)$ as a consequence of the maximum principle. Without appropriate data restrictions, the solution of (1) may blow up at some finite time $t^{*}[1,3]$. The goal of this paper is to determine conditions on the data and on the geometry of $\Omega$ sufficient to insure that the solution remains bounded for all time and to establish exponential decay (in time) bounds for $u(\mathbf{x}, t)$ and $|\nabla u|$ under these conditions.

L.E. Payne: Department of Mathematics, Cornell University, Ithaca, N.Y, 14853, USA; lep@cornell.edu

G.A. Philippin: Département de mathématiques et de statistique, Université Laval, Québec, Canada, G1K 7P4; gphilip@mat.ulaval.ca

ISSN 0232-2064 / \$ 2.50 (c) Heldermann Verlag Berlin 
The particular case $D(\mathbf{x})=1$ has already been investigated in an earlier paper [5] via a maximum principle that we are going to modify in order to cover the more general case $D(\mathbf{x}) \neq 1$.

The notations $u_{, i}:=\frac{\partial u}{\partial x_{i}}$ and $u_{, i k}:=\frac{\partial^{2} u}{\partial x_{i} \partial x_{k}}$ will be used in the remainder of the paper, and summation from 1 to $N$ over repeated indices will be assumed. Using these notations, we have for instance $u_{, i} u_{, i}=\sum_{i=1}^{N}\left(\frac{\partial u}{\partial x_{i}}\right)^{2}=|\nabla u|^{2}$.

\section{A modified version of the maximum principle}

The following lemmas are basic in our investigation.

Lemma 2.1. Let $u(\mathbf{x}, t)$ be a classical solution of the parabolic PDE

$$
\nabla(D \nabla u)-u_{, t}=-f(u), \quad \mathbf{x} \in \Omega \subset \mathbb{R}^{N}, t \in(0, \tau),
$$

where $f$ is a differentiable function assumed to satisfy the conditions

$$
f(s) \geq 0, f(0)=0, f^{\prime}(s) \geq 0, \frac{f(s)}{s} \text { non-decreasing for } s>0,
$$

and $D \in C^{2}(\Omega)$ is assumed to satisfy the condition $D(\mathbf{x}) \geq D_{0}>0$ for $\mathbf{x} \in \Omega$. Let $\Phi(\mathbf{x}, t)$ be the auxiliary function defined as

$$
\Phi(\mathbf{x}, t):=\left\{|\nabla u|^{2}+\alpha \beta u^{2}+2 \beta F(u)\right\} e^{2 \alpha t}
$$

with $F(u):=\int_{0}^{u} f(s) d s$. In (3), $\alpha$ and $\beta$ are parameters subject to the restrictions

$$
\begin{aligned}
& \alpha \geq \alpha_{0}:=\frac{\epsilon}{1-\epsilon} \max _{\Omega}\left\{\frac{N|\nabla D|^{2}}{4 D(1-\epsilon)}+\sqrt{D_{, i k} D_{, i k}}\right\} \\
& \beta \geq \beta_{0}:=\frac{1}{\epsilon D_{0}}
\end{aligned}
$$

for some constant $\epsilon \in(0,1)$. Then the function $\Phi(\mathbf{x}, t)$ satisfies a parabolic partial differential inequality of the form

$$
L \Phi:=\nabla(D \nabla \Phi)-\Phi_{, t}+\cdots \geq 0 \quad, \quad(\mathbf{x}, t) \in \Omega \times[0, \tau] \backslash \omega,
$$

where $\omega:=\{(\mathbf{x}, t) \in \Omega \times(0, \tau) \mid \nabla u(\mathbf{x}, t)=0\}$ is the set of critical points of $u$. In (5) dots stand for terms containing first order derivatives of $\Phi$.

Complement to Lemma 2.1: Suppose in addition that the function $D$ is concave in $\Omega$, then Lemma 2.1 holds with

$$
\alpha_{0}:=\frac{N \epsilon}{4(1-\epsilon)^{2}} \max _{\Omega} \frac{|\nabla D|^{2}}{D} .
$$


Proof. We compute successively

$$
\begin{aligned}
\Phi_{, k}= & 2\left\{u_{, i k} u_{, i}+\alpha \beta u u_{, k}+\beta f u_{, k}\right\} e^{2 \alpha t} \\
\nabla(D \nabla \Phi)=2\{ & \left\{D u_{, i k} u_{, i k}+\left(D u_{, i k}\right)_{, k} u_{, i}+D \beta\left(\alpha+f^{\prime}\right)|\nabla u|^{2}\right. \\
& +\beta(\alpha u+f) \nabla(D \nabla u)\} e^{2 \alpha t} .
\end{aligned}
$$

Differentiating equation (1), we obtain $\left(D_{, i} u_{, k}+D u_{, k i}\right)_{, k}=\left(D u_{, k}\right)_{, k i}=u_{, t i}-$ $f^{\prime} u_{, i}$, from which we compute

$$
\left(D u_{, i k}\right)_{, k} u_{, i}=u_{, t i} u_{, i}-f^{\prime}|\nabla u|^{2}-D_{, i k} u_{, i} u_{, k}-\Delta u \nabla D \nabla u .
$$

Inserting (9) in (8) and making use of (1), we obtain

$$
\begin{aligned}
\nabla(D \nabla \Phi)=2 & \left\{D u_{, i k} u_{, i k}+\left[f^{\prime}(\beta D-1)+\alpha \beta D\right]|\nabla u|^{2}\right. \\
& +\beta(\alpha u+f)\left(u_{, t}-f\right) \\
& \left.+u_{, i t} u_{, i}-D_{, i k} u_{, i} u_{, k}-\Delta u \nabla u \nabla D\right\} e^{2 \alpha t} .
\end{aligned}
$$

Moreover, we compute

$$
\Phi_{, t}=2\left\{u_{, i t} u_{, i}+\beta u_{, t}(\alpha u+f)+\alpha|\nabla u|^{2}+\alpha^{2} \beta u^{2}+2 \alpha \beta F\right\} e^{2 \alpha t} .
$$

Combining (10) and (11), we obtain

$$
\begin{array}{rl}
\nabla(D \nabla \Phi)-\Phi_{, t}=2 & 2\left\{D u_{, i k} u_{, i k}+(D \beta-1)\left(\alpha+f^{\prime}\right)|\nabla u|^{2}-\beta f(\alpha u+f)\right. \\
& \left.-D_{, i k} u_{, i} u_{, k}-\Delta u \nabla u \nabla D-\alpha^{2} \beta u^{2}-2 \alpha \beta F\right\} e^{2 \alpha t} .
\end{array}
$$

We now combine the following two inequalities

$$
\begin{aligned}
& u_{, i k} u_{, i k} \geq|\nabla u|^{-2} u_{, i k} u_{, k} u_{, i j} u_{, j}=\beta^{2}(\alpha u+f)^{2}+\cdots \\
& u_{, i k} u_{, i k} \geq \frac{(\Delta u)^{2}}{N}
\end{aligned}
$$

to obtain

$$
u_{, i k} u_{, i k} \geq \epsilon \beta^{2}(\alpha u+f)^{2}+\frac{1-\epsilon}{N}(\Delta u)^{2}+\cdots
$$

valid for arbitrary $\epsilon \in[0,1]$, where dots stand for terms containing $\Phi_{, k}$. We note that the last equality in (13) follows from (7) rewritten as $u_{, i k} u_{, k}=-\beta(\alpha u+$ f) $u_{, k}+\cdots$ for $k=1, \ldots, N$. From assumption (2), we have

$$
f(s) \leq \frac{f(u)}{u} s \quad, \quad 0 \leq s \leq u .
$$

Integrating both sides of (15) from 0 to $u$, we obtain the inequality

$$
F(u):=\int_{0}^{u} f(s) d s \leq \frac{1}{2} u f(u) .
$$


Inserting (14) and (16) in (12), we obtain after some reduction

$$
\begin{aligned}
L \Phi:= & \nabla(D \nabla \Phi)-\Phi_{, t}+\cdots \\
\geq 2 & \left\{\beta(D \epsilon \beta-1)(\alpha u+f)^{2}+\frac{D(1-\epsilon)}{N}(\Delta u)^{2}\right. \\
& \left.\quad-\Delta u \nabla u \nabla D+(D \beta-1)\left(\alpha+f^{\prime}\right)|\nabla u|^{2}-D_{, i k} u_{, i} u_{, k}\right\} e^{2 \alpha t} .
\end{aligned}
$$

Finally, we make use of the inequalities

$$
\begin{aligned}
\frac{D(1-\epsilon)}{N}(\Delta u)^{2}-\Delta u \nabla u \nabla D & =\frac{D(1-\epsilon)}{N}\left[\Delta u-\frac{N \nabla u \nabla D}{2 D(1-\epsilon)}\right]^{2}-\frac{N(\nabla u \nabla D)^{2}}{4 D(1-\epsilon)} \\
& \geq-\frac{N(\nabla D \nabla u)^{2}}{4 D(1-\epsilon)} \\
& \geq-\frac{N|\nabla D|^{2}}{4 D(1-\epsilon)}|\nabla u|^{2},
\end{aligned}
$$

and $D_{, i k} u_{, i} u_{, k} \leq \sqrt{D_{, i k} D_{, i k}}|\nabla u|^{2}$ to obtain the differential inequality

$$
\begin{aligned}
L \Phi \geq 2 & \left\{\beta(D \epsilon \beta-1)(f+\alpha u)^{2}\right. \\
& \left.+\left[\alpha(D \beta-1)-\frac{N|\nabla D|^{2}}{4 D(1-\epsilon)}-\sqrt{D_{, i k} D_{, i k}}\right]|\nabla u|^{2}\right\} e^{2 \alpha t} .
\end{aligned}
$$

With $\beta \geq \beta_{0}:=\frac{1}{\epsilon D_{0}}$, we obtain

$$
L \Phi \geq 2\left\{\frac{\alpha(1-\epsilon)}{\epsilon}-\frac{N|\nabla D|^{2}}{4 D(1-\epsilon)}-\sqrt{D_{, i k} D_{, i k}}\right\}|\nabla u|^{2} e^{2 \alpha t} \geq 0,
$$

valid for $\alpha \geq \alpha_{0}$ defined in (4). Moreover if $D$ is concave in $\Omega$, we may drop the term $D_{, i k} u_{, i} u_{, k}$ in (17), so that $\alpha_{0}$ reduces to (6).

We note that if $D$ is constant in $\Omega$, the inequality $L \Phi \geq 0$ directly follows from (17) with $\epsilon:=1, \beta \geq D^{-1}, \alpha \geq 0$.

As a direct application of Nirenberg's maximum principle [4, 6], we conclude then that under the assumptions of Lemma 2.1, $\Phi$ defined in (3) takes its maximum value either

(i) at a point $\hat{\mathbf{P}}:=(\hat{\mathbf{x}}, \hat{t})$ with $\hat{\mathbf{x}} \in \partial \Omega$, or

(ii) at a point $\overline{\mathbf{P}}:=(\overline{\mathbf{x}}, \bar{t}) \in \omega$, or

(iii) at a point $\tilde{\mathbf{P}}:=(\tilde{\mathbf{x}}, 0), \tilde{\mathbf{x}} \in \Omega$. 
The next Lemma shows that the first possibility (i) implies (iii) under some appropriate conditions.

Lemma 2.2. Assume that $u(\mathbf{x}, t)$ is the classical solution of problem (1), and that $D(\mathbf{x})$ satisfies the following boundary condition

$$
(N-1) K D+\frac{\partial D}{\partial n} \geq 0, \quad \mathbf{x} \in \partial \Omega,
$$

with $\partial \Omega \in C^{2+\delta}$, where $K$ is the average curvature of $\partial \Omega$ and $\frac{\partial D}{\partial n}$ is the outward normal derivative of $D$. Then the maximum value of $\Phi$ cannot occur at $\hat{\mathbf{P}}$ unless the equality sign in (18) holds there, in which case the maximum will also occur at $\tilde{\mathbf{P}}$.

Proof. We compute the outward normal derivative of $\Phi$ on $\partial \Omega$

$$
\frac{\partial \Phi}{\partial n}=2 u_{n} u_{n n} e^{2 \alpha t}, \quad \mathbf{x} \in \partial \Omega .
$$

Since $\partial \Omega \in C^{2+\delta}$, the differential equation (1) is satisfied on $\partial \Omega$. Using normal coordinates, we compute from (1)

$$
D \Delta u+\nabla D \nabla u=D\left\{u_{n n}+(N-1) K u_{n}\right\}+\nabla D \nabla u=0, \quad \mathbf{x} \in \partial \Omega,
$$

from which we obtain

$$
u_{n n}=-(N-1) K u_{n}-\frac{1}{D} \nabla D \nabla u, \quad \mathbf{x} \in \partial \Omega .
$$

Combining (19) and (20), we obtain

$$
\frac{\partial \Phi}{\partial n}=-\frac{2}{D}\left\{(N-1) K D+\frac{\partial D}{\partial n}\right\}|\nabla u|^{2} e^{2 \alpha t} \leq 0, \quad \mathbf{x} \in \partial \Omega
$$

in view of assumption (18), so that the maximum of $\Phi$ could occur at $\hat{\mathbf{P}}$ only if $\frac{\partial \Phi}{\partial n}=0$ there. But from Friedman's maximum principle $[2,6]$, we would then conclude that $\Phi=$ const in $\Omega \times(0, \hat{t})$, so that $\Phi$ would already take its maximum initially.

\section{Application to the linear case}

The goal of this section is to derive explicit decay bounds for the solution $u(\mathbf{x}, t)$ of problem (1) in the linear case $f=0$. As a consequence of Lemmas 2.1 and 2.2 , we know that the auxiliary function

$$
\Phi(\mathbf{x}, t):=\left\{|\nabla u|^{2}+\alpha \beta_{0} u^{2}\right\} e^{2 \alpha t}
$$


with $\beta_{0}=\frac{1}{\epsilon D_{0}}, \alpha \geq \alpha_{0}$, takes its maximum either at a critical point $(\overline{\mathbf{x}}, \bar{t})$, or initially. Suppose now that $\Phi$ takes its maximum at a critical point $(\overline{\mathbf{x}}, \bar{t})$, i.e. suppose we have

$$
|\nabla u(\mathbf{x}, \bar{t})|^{2} \leq \alpha \beta_{0}\left[u_{m}^{2}-u^{2}(\mathbf{x}, \bar{t})\right], \quad \mathbf{x} \in \Omega,
$$

with $u_{m}:=\max _{\Omega \times[0, \infty)} u<\infty$. Rewriting inequality (21) as

$$
\frac{|d u(\mathbf{x}, \bar{t})|}{\sqrt{u_{m}^{2}-u^{2}(\mathbf{x}, \bar{t})}} \leq \sqrt{\alpha \beta_{0}} d|\mathbf{x}|
$$

and integrating (22) from $\overline{\mathbf{x}}$ to the nearest point $\mathbf{x}^{*}$ on $\partial \Omega$, we obtain

$$
\frac{\pi}{2} \leq \sqrt{\alpha \beta_{0}}\left|\overline{\mathbf{x}}-\mathbf{x}^{*}\right| \leq \sqrt{\alpha \beta_{0}} d
$$

where $d$ is the radius of the largest ball contained in $\Omega$, i.e.

$$
\alpha \beta_{0} \geq \frac{\pi^{2}}{4 d^{2}}
$$

Inequality (23) is a necessary condition for $\Phi$ to take its maximum value at a critical point. Clearly if (23) is violated, i.e. if we have $\left(\alpha_{0} \beta_{0} \leq\right) \alpha \beta_{0}<\frac{\pi^{2}}{4 d^{2}}$, the auxiliary function $\Phi$ cannot take its maximum at a critical point $(\overline{\mathbf{x}}, \bar{t})$, so that the maximum must occur initially. This leads to the explicit decay bounds

$$
|\nabla u|^{2}+\alpha \beta_{0} u^{2} \leq G^{2} e^{-2 \alpha t}
$$

with

$$
G^{2}:=\max _{\Omega}\left\{|\nabla g|^{2}+\alpha \beta_{0} g^{2}\right\}
$$

Letting $\alpha \rightarrow \frac{\pi^{2}}{4 \beta_{0} d^{2}}$ in (24), (25), we obtain the inequality

$$
|\nabla u|^{2}+\frac{\pi^{2}}{4 d^{2}} u^{2} \leq \Gamma^{2} \exp \left(-\frac{\pi^{2} \epsilon D_{0}}{2 d^{2}} t\right)
$$

with

$$
\Gamma^{2}:=\max _{\Omega}\left\{|\nabla g|^{2}+\frac{\pi^{2}}{4 d^{2}} g^{2}\right\}
$$

We note that the above computation makes sense only if we have

$$
\alpha_{0}:=\frac{\epsilon}{1-\epsilon} \max _{\Omega}\left\{\frac{N|\nabla D|^{2}}{4 D(1-\epsilon)}+\sqrt{D_{, i k} D_{, i k}}\right\} \leq \frac{\pi^{2}}{4 d^{2} \beta_{0}}=\frac{\pi^{2} \epsilon D_{0}}{4 d^{2}},
$$

for some $\epsilon \in(0,1)$. 


\section{Application to the nonlinear case}

In the nonlinear case, $f \not \equiv$ const. it is well known that the solution $u(\mathbf{x}, t)$ may blow up at some finite time $t^{*}$. In this section, we use Lemmas 2.1 and 2.2 and establish some conditions on the data of problem (1) in order to constrain its solution $u(\mathbf{x}, t)$ to exist for all time. Moreover, we shall prove that $u$ and $|\nabla u|$ decay exponentially in time under appropriate restrictions on the data of problem (1). Our investigation makes use of the following estimate.

Lemma 4.1. Let $u(\mathbf{x}, t)$ be the solution of problem (1) where $f(s)$ satisfies (2). Then if $\tau$ is any time prior to blow-up time $t^{*}$, there holds the estimate

$$
0 \leq u(\mathbf{x}, t) \leq U \exp \left\{-\left(\frac{\pi^{2}}{4 \beta_{0} d^{2}}-\mu\right) t\right\}, \quad \mathbf{x} \in \Omega, 0 \leq t \leq \tau
$$

with

$$
\mu:=\frac{f\left(u_{m}\right)}{u_{m}}, \quad u_{m}:=\max _{\Omega \times[0, \tau]} u(\mathbf{x}, t)
$$

and

$$
U:=\max _{\Omega} \sqrt{g^{2}+\frac{4 d^{2}}{\pi^{2}}|\nabla g|^{2}}
$$

Proof. We introduce the auxiliary function $v(\mathbf{x}, t)$ defined as

$$
u(\mathbf{x}, t)=v(\mathbf{x}, t) \exp (\mu t),
$$

and we compute

$$
\begin{aligned}
{\left[\nabla(D \nabla v)-v_{, t}\right] \exp (\mu t) } & =\nabla(D \nabla u)-u_{, t}+u \frac{f\left(u_{m}\right)}{u_{m}} \\
& \geq \nabla(D \nabla u)-u_{, t}+f(u)=0,
\end{aligned}
$$

since we have $\frac{f\left(u_{m}\right)}{u_{m}} \geq \frac{f(u)}{u}$ in view of $(2)$. We obtain

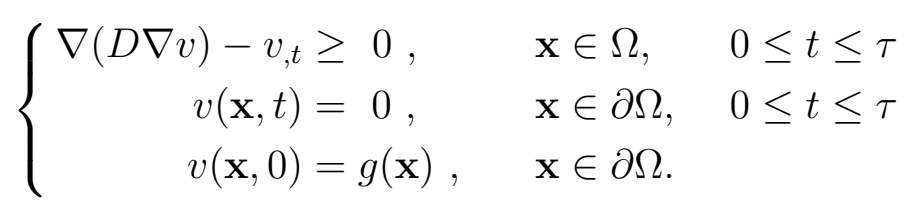

From a standard comparison theorem [6], we have $v(\mathbf{x}, t) \leq w(\mathbf{x}, t)$ for $\mathbf{x} \in \Omega$ and $0 \leq t \leq \tau$, where $w(\mathbf{x}, t)$ is the solution of (1) with $f=0$. By making use of (26), (27), we obtain

$$
w(\mathbf{x}, t) \leq U \exp \left(-\frac{\pi^{2}}{4 \beta_{0} d^{2}} t\right)
$$

where $U$ is defined in (29). Combining (30) and (31), we obtain the desired upper bound (28). 
Lemma 4.2 will be used to establish the following result.

Lemma 4.2. Let $u(\mathbf{x}, t)$ be the solution of problem (1). Let $f(s)$ satisfy (2). Assume moreover that the initial data is small enough in the sense

$$
\frac{f(U)}{U}<\frac{\pi^{2}}{4 \beta_{0} d^{2}}=: \gamma_{0}
$$

where $U, \beta_{0}$, and $d$ have the same meaning as in Lemma 4.1. Then $t^{*}=\infty$, i.e. $u(\mathbf{x}, t)$ does not blow up in finite time. Moreover, we have

$$
\max _{\Omega} \frac{f(u(\mathbf{x}, t))}{u(\mathbf{x}, t)}<\gamma_{0}, \quad 0 \leq t<\infty .
$$

Proof. We consider two possibilities.

First case: $\mu \leq \gamma_{0}$. In this case, we conclude from (28) that $0 \leq u(\mathbf{x}, t) \leq$ $U$ for $\mathbf{x} \in \Omega$ and $0 \leq t<\infty$.

Second case: $\mu>\gamma_{0}$. In this case, we suppose that $u(\mathbf{x}, t)$ blows up at finite time $t^{*}$. Then by continuity there exists a first time $\tau$ at which we have

$$
\max _{\Omega} \frac{f(u(\mathbf{x}, \tau))}{u(\mathbf{x}, \tau)}=\gamma_{0} .
$$

It then follows from Lemma 4.1 that $u(\mathbf{x}, \tau) \leq U$ for $\mathbf{x} \in \Omega$, from which we obtain

$$
\frac{f(u(\mathbf{x}, \tau))}{u(\mathbf{x}, \tau)} \leq \frac{f(U)}{U}<\gamma_{0}, \quad \mathbf{x} \in \Omega,
$$

thanks to (2) and (28). We are then led to the inequality

$$
\max _{\Omega} \frac{f(u(\mathbf{x}, \tau))}{u(\mathbf{x}, \tau)}<\gamma_{0}
$$

in contradiction to the definition of $\tau$.

Under the assumptions of Lemmas 2.1, 2.2, 4.1 and 4.2, we know that the solution $u(\mathbf{x}, t)$ of $(1)$ exists and remains bounded for all time. Moreover the auxiliary function $\Phi$ with $\alpha \geq \alpha_{0}, \beta=\beta_{0}$, defined for all time in (3), takes its maximum either at a critical point $\overline{\mathbf{P}}:=(\overline{\mathbf{x}}, \bar{t})$ or initially. We shall see that if the parameter $\alpha\left(\geq \alpha_{0}\right)$ is small enough, $\Phi$ must assume its maximum value initially, which implies that $u(\mathbf{x}, t)$ and $|\nabla u|$ decay exponentially in time.

Lemma 4.3. Assume that $g(\mathbf{x})$ is small enough in the following sense

$$
\frac{f(U)}{U}<\frac{\pi^{2}}{4 \beta_{0} d^{2}}-\alpha=\gamma_{0}-\alpha
$$

for some $\alpha \geq \alpha_{0}$, where $\alpha_{0}, \beta_{0}, U$, $d$ have already been defined. Then the function $\Phi$ cannot take its maximum value at a critical point of $u$. 
Proof. We assume that $\Phi$ takes its maximum value at a critical point $\overline{\mathbf{P}}:=$ $(\overline{\mathbf{x}}, \bar{t})$, i.e. that we have $\Phi(\mathbf{x}, t) \leq \Phi(\overline{\mathbf{x}}, \bar{t}), \mathbf{x} \in \Omega, t>0$. Evaluated at $t=\bar{t}$, this inequality may be rewritten as

$$
|\nabla u|^{2} \leq \alpha \beta_{0}\left[u_{m}^{2}-u^{2}(\mathbf{x}, \bar{t})\right]+2 \beta_{0}\left[F\left(u_{m}\right)-F(u(\mathbf{x}, \bar{t}))\right]
$$

with $u_{m}:=\max _{\Omega \times[0, \infty)} u(\mathbf{x}, t)$. Using the generalised mean value theorem and (2), we obtain

$$
\begin{aligned}
2\left[F\left(u_{m}\right)-F(u(\mathbf{x}, \bar{t}))\right] & =\frac{2\left[F\left(u_{m}\right)-F(u(\mathbf{x}, \bar{t}))\right]}{u_{m}^{2}-u^{2}(\mathbf{x}, \bar{t})}\left[u_{m}^{2}-u^{2}(\mathbf{x}, \bar{t})\right] \\
& =\frac{f(\xi)}{\xi}\left[u_{m}^{2}-u^{2}(\mathbf{x}, \bar{t})\right] \\
& \leq \frac{f\left(u_{m}\right)}{u_{m}}\left[u_{m}^{2}-u^{2}(\mathbf{x}, \bar{t})\right],
\end{aligned}
$$

where $\xi$ is some intermediate value in $\left(u, u_{m}\right)$. From (35) and (36), we obtain the inequality

$$
|\nabla u(\mathbf{x}, \bar{t})|^{2} \leq \beta_{0}\left[\alpha+\frac{f\left(u_{m}\right)}{u_{m}}\right]\left(u_{m}^{2}-u^{2}(\mathbf{x}, \bar{t})\right), \quad \mathbf{x} \in \Omega .
$$

Rewriting the above inequality in the form

$$
\frac{|d u(\mathbf{x}, \bar{t})|}{\sqrt{u_{m}^{2}-u^{2}(\mathbf{x}, \bar{t})}} \leq \sqrt{\beta_{0}\left(\alpha+\frac{f\left(u_{m}\right)}{u_{m}}\right)} d|\mathbf{x}|,
$$

and integrating along a straight line segment from the critical point $\overline{\mathbf{x}}$ to the nearest boundary point $\mathbf{x}^{*}$, we obtain the inequality

$$
\alpha+\frac{f\left(u_{m}\right)}{u_{m}} \geq \frac{\pi^{2}}{4 d^{2} \beta_{0}}=\gamma_{0}
$$

where $d$ is the inradius of $\Omega$. Inequality (37) is a necessary condition on $\alpha$ to be satisfied for $\Phi$ to take its maximum value at a critical point. To establish the conclusion of Lemma 4.3, it remains to show that (37) is violated if (34) is satisfied. Indeed, (34) implies the inequality $\frac{f(U)}{U}<\gamma_{0}$. It then follows from (33) that $\frac{f\left(u_{m}\right)}{u_{m}}<\gamma_{0}$, and from Lemma 4.1 that $u_{m}<U$, so that we have in view of (2) and (34)

$$
\frac{f\left(u_{m}\right)}{u_{m}} \leq \frac{f(U)}{U}<\gamma_{0}-\alpha
$$

in contradiction to $(37)$.

The result of this paper may be sumarised as follows. 
Theorem 4.4. Let $u(\mathbf{x}, t)$ be the solution of (1) with $f(s)$ satisfying the conditions (2). Assume that $D(\mathbf{x})$ in (1) satisfies the conditions

$$
\begin{aligned}
D(\mathbf{x}) & \geq D_{0}>0, & & \mathbf{x} \in \Omega \\
(N-1) K D+\frac{\partial D}{\partial n} & \geq 0, & & \mathbf{x} \in \partial \Omega,
\end{aligned}
$$

where $K$ is the average curvature of $\partial \Omega$. Assume that the initial data are compatible with the inequalities

$$
\alpha_{0} \leq \alpha \leq \gamma_{0}-\frac{f(U)}{U}=: \alpha_{1}
$$

where

$$
\begin{aligned}
\gamma_{0} & :=\frac{\pi^{2}}{4 d^{2} \beta_{0}}=\frac{\pi^{2} D_{0}}{4 d^{2} \epsilon} \\
U & :=\max _{\Omega} \sqrt{|\nabla g|^{2}+\frac{\pi^{2}}{4 d^{2}} g^{2}}
\end{aligned}
$$

for some $\epsilon \in(0,1)$. Then the decay estimate

$$
|\nabla u|^{2}+\alpha_{1} \beta_{0} u^{2}+2 \beta_{0} F(u) \leq \Lambda \exp \left(-2 \alpha_{1} t\right)
$$

with $\Lambda:=\max _{\Omega}\left\{|\nabla g|^{2}+\alpha_{1} \beta_{0} g^{2}+2 \beta_{0} F(g)\right\}$ holds.

\section{References}

[1] Ball, J. M.: Remarks on blow-up and nonexistence theorems for nonlinear evolution equations. Quart. J. Math. Oxford 28 (1977), 473 - 486.

[2] Friedman, A.: Remarks on the maximum principle for parabolic equations and its applications. Pacific J. Math. 8 (1958), $201-211$.

[3] Kielhöfer, H.: Halbgruppen und semilineare Anfangs-Randwert-Probleme. Manuscripta Math. 12 (1974), 121 - 152.

[4] Nirenberg, L.: A strong maximum principle for parabolic equations. Comm. Pure Appl. Math. 6 (1953), 167 - 177.

[5] Payne, L. E. and G. A. Philippin: Decay bounds for solutions of second order parabolic problems and their derivatives. Math. Models and Meth. in Appl. Sci. 5 (1995), $95-110$.

[6] Protter, M. H. and H. F. Weinberger: Maximum Principles in Differential Equations. Englewood Cliffs, N.J.: Prentice-Hall Inc. 1967.

Received 01.12.2003 